\title{
Comparative fluorescence analysis of the bovine sperm using IVA-520 (anti-CD46 antibody) and lectins: probable localisation of CD46 on bovine sperm membrane
}

\author{
Jana Jankovicova, Jana Antalikova, Michal Simon, Katarina Michalkova and Lubica Horovska \\ Institute of Animal Biochemistry and Genetics, Centre of Excellence of Slovak Research and Development Agency „Biomem- \\ branes 2008", Slovak Academy of Sciences, Ivanka pri Dunaji, Slovak Republic
}

\begin{abstract}
Membrane cofactor protein (CD46) is complement regulatory protein with probable function in the reproduction process. Expression of CD46 on human, mice, rat and guinea pig spermatozoa is restricted to the inner acrosomal membrane. In spite of the presence of anti-sperm antibodies and other potential complement activating agents in follicular fluid, CD46 is not expressed on the plasma membrane of spermatozoa as the other complement regulatory proteins (DAF and CD59) in human. Using dual immunofluorescence labelling with mAb IVA-520 (anti-bovine CD46) and various lectins with different binding pattern or monoclonal antibody ACR.4, targeted against intra-acrosomal protein, we excluded the expression of CD46 on the inner acrosomal membrane as well as in the acrosomal content but, we suggested the localization of this molecule on the outer acrosomal membrane and possibly on the plasma membrane of bovine sperm.
\end{abstract}

Key words: Sperm membrane - Bovine CD46 - Lectins - MAb IVA-520

\begin{abstract}
Abbreviations: ConA, Canavalia ensiformis agglutinin; DAF, decay accelerating factor; MCP, membrane cofactor protein; PNA, Arachis hypogaea agglutinin; PSA, Pisum sativum agglutinin; WGA, Triticum vulgaris agglutinin.
\end{abstract}

\section{Introduction}

The CD46 (MCP; membrane cofactor protein) is a widely distributed membrane bound regulatory protein that inhibits complement activation on host cells (Seya et al.1986; Ballard et al. 1988; Seya and Atkinson 1989; Oglesby et al. 1992). On spermatozoa in human (Anderson et al. 1989, Fenichel 1990), mice (Inoue et al. 2003), rats (Mizuno et al. 2004) and probably in guinea pigs (Hosokawa et al. 1996), CD46 is restricted to the inner acrosomal membrane and was not found on the plasma membrane. This has led to speculation that CD46 may be involved in sperm-egg binding, independent of its role as complement activation. Anderson et al. (1993) described a model whereby MCP might be one half of a C3b dimmer bridge between the spermatozoa and the

Correspondence to: Jana Antalikova, Institute of Animal Biochemistry and Genetics, Slovak Academy of Sciences, Moyzesova 61, 90028 Ivanka pri Dunaji, Slovak Republic

E-mail: Jana.Antalikova@savba.sk egg. The expression of complement receptors 1 and 3 on the plasma membrane of human eggs supports this possibility (Anderson et al. 1993). MCP's complement regulatory activity could protect acrosome reacted spermatozoa during the final step of fertilization in as much as the concentration of complement components in follicular fluid is equivalent to that in serum (Clarke et al. 1984; Pericone et al. 1990, 1992; Vanderpuye et al. 1992) and acrosin, a protease released during the acrosome reaction can cleave C3 (Anderson et al. 1993). According to Riley et al. (2005), with presence of the anti-sperm antibodies and other potential complement activating agents in follicular fluid, it is curious that MCP is not also expressed on the plasma membrane of spermatozoa as are two other complement regulatory proteins, decay accelerating factor (DAF) (Cervoni et al. 1993) and CD59 (Fenichel et al. 1994). It is interesting that in the transgenic mouse, human CD46 was also expressed on the plasma membrane of spermatozoa (Riley et al. 2002).

In our recent paper, monoclonal antibody (mAb IVA-520) against bovine CD46 molecule has been described and its 
reactivity on sperm cells has been evidenced (Antalíková et al. 2007). The previous experiments (immunofluorescence testing, immunoprecipitation of biotinylated surface sperm molecules) revealed the presence of CD46 on the plasma membrane, acrosomal membrane or in the acrosomal content, respectively. To verify these findings, bovine sperm were treated with mAb IVA-520 and lectins (Pisum sativum agglutinin (PSA); Arachis hypogaea agglutinin (PNA); Triticum vulgaris agglutinin (WGA); Canavalia ensiformis agglutinin (ConA)) with different binding pattern or monoclonal antibody ACR.4 targeted against intra-acrosomal protein (Pěknicová et al. 1998) and assayed in immunofluorescence test.

\section{Material and Methods}

\section{Sperm}

Frozen-thawed bovine sperm obtained from Slovak Breeding Services (Lužianky, Slovak Republic) were used for all experiments. Frozen sperm were thawed in phosphate buffered saline (PBS), washed twice and centrifuged at $250 \times g$ at room temperature. Either frozen-thawed or sperm stimulated to acrosome exocytosis were tested.

\section{Acrosome reaction}

Briefly, fraction of motile and viable sperm was separated using a swim-up for $10 \mathrm{~min}$ at $39^{\circ} \mathrm{C}$ and $5 \% \mathrm{CO}_{2}$ and washed in Sp-Tl medium (Minitübe, GmbH, Germany) supplemented with BSA V-fraction, natrium pyruvate and gentamycine (Makarevich and Markkula 2002). Acrosome reaction was induced by calcium ionophore A23 187 (Sigma, St. Louis, $\mathrm{MO})(1 \mu \mathrm{mol} / \mathrm{l})$ for $1 \mathrm{~h}$ at $39^{\circ} \mathrm{C}$ and $5 \% \mathrm{CO}_{2}$. Both acrosomes reacted and non-reacted sperm were stained with Hoechst H33 342 (Sigma, St. Louis, USA) $(8 \mu \mathrm{g} / \mathrm{ml})$. Subsequently, the cell smears were prepared and "antibody-antibody assay" or "antibody-lectin assay" were applied.

\section{Antibodies}

The mouse mAb IVA-520 against bovine CD46 molecule (Antalíková et al. 2007) purified on protein A-Sepharose and conjugated with Fluorescein Isothiocyanate (FITC) according to the manufacturer's protocol (EZ-Label ${ }^{\mathrm{TM}}$ Fluorescein Isothiocyanate Protein Labelling Kit, Pierce, Rockford, USA) was used. The mAb ACR.4 targeted against intraacrosomal protein (Pěknicová et al. 1998) was purchased from Exbio (Prague, Czech Republic). As the secondary antibody the Goat anti mouse IgG-Tetramethyl Rhodamine Isothiocyanate (TRITC) or Goat anti mouse IgG-FITC were applied.

\section{Immunofluorescence assay}

Intact frozen-thawed sperm were tested by indirect immunofluorescence. Samples were treated with mAb IVA-520FITC-conjugate. Both IVA-520-reacted and non-reacted sperm were stained with Hoechst 33342 (Sigma, St. Louis, USA). Subsequently, the cell smears were prepared and evaluated.

For permeabilisation of sperm membranes, the slides with sperm smears were shortly (30 s) dipped into 100\% methanol $-20^{\circ} \mathrm{C}$, washed and blocked with Super Block buffer (Pierce, Rockford, USA). All treatments were applied in a humid chamber to prevent drying of the cell smears.

Firstly, in the "antibody-antibody assay" mAb IVA-520 and mAb ACR.4 were applied. Briefly, after treatment with mAb ACR.4 and incubation with Goat anti mouse IgGTRITC the samples were treated with mAb IVA-520-FITC conjugate.

Secondly, in the "antibody-lectin assay" mAb IVA-520 and lectins combination were applied. The sperm were treated with primary antibody mAb IVA-520, for $1 \mathrm{~h}$ at $37^{\circ} \mathrm{C}$ and after washing in PBS, the secondary antibody IgG-TRITC was applied. The slides were then treated with fluoresceinated lectin probe (PSA conjugated with FITC (ICN Biomedicals, Irvin, USA)) or the sperm was treated with primary antibody $\mathrm{mAb}$ IVA-520-FITC, for $1 \mathrm{~h}$ at $37^{\circ} \mathrm{C}$ and after washing in PBS, PNA, WGA and ConA, all rhodamine conjugates (Vector Laboratories, Burlingame, USA) were used. Finally, the smears were thoroughly washed and mounted in $90 \%$ glycerol in PBS, pH 9.0, and the slides were evaluated under a Leica DM5500 B epifuorescence microscope at 40× magnification and fluorescence images were recorded using a Leica DFC340 FX digital camera and processed using Leica Advanced Fluorescence software.

\section{Statistical analysis}

For all specimens, staining patterns were quantified by scoring a minimum of 200 sperm for each assay. Each experiment was repeated at least three times. Percentage scores were calculated to normalise the data. Significant differences were identified by performing Student's $t$-test using the statistical software Sigma Plot 10.0.

\section{Results}

Reaction pattern of IVA-520 on intact and frozen-thawed sperm

In indirect immunofluorescence assay, $46 \%$ reactivity of intact frozen-thawed sperm has been denoted (Fig. 1). After permeabilisation of sperm membranes, a number of labeled 
Table 1. Reactivity of mAb IVA-520 on frozen-thawed sperm in suspension, permeabilised and acrosome-reacted bovine sperm

\begin{tabular}{|c|c|c|c|}
\hline $\begin{array}{l}\text { Reaction } \\
\text { pattern }\end{array}$ & $\begin{array}{l}\text { Frozen-thawed } \\
\text { intact sperm }\end{array}$ & $\begin{array}{c}\text { Frozen-thawed } \\
\text { permeabilized } \\
\text { sperm }\end{array}$ & $\begin{array}{l}\text { Acrosome-reacted } \\
\text { permeabilized } \\
\text { sperm }\end{array}$ \\
\hline 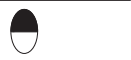 & $46.16 \pm 3.89^{*}$ & $89.02 \pm 3.76^{*}$ & $75.37 \pm 3.49$ \\
\hline$\theta$ & - & - & $2.93 \pm 5.43$ \\
\hline & $53.84 \pm 3$ & $10.98 \pm 3.76^{\star}$ & $21.68 \pm 3.46$ \\
\hline \multicolumn{4}{|c|}{$\begin{array}{l}\text { The values are presented as } \% \text { mean } \pm \mathrm{SD}(n=20) ;{ }^{*} p<0.01 \text {. } \\
\text { population of sperm stained in the anterior part of the head } \\
\text { hroughout the acrosomal region; } \theta \text { population of sperm with } \\
\text { taining limited to the equatorial acrosomal segment; } 0 \text { population } \\
\text { f completely unstained sperm. }\end{array}$} \\
\hline
\end{tabular}

cells increased to $89 \%$. In both experiments, a selective staining of the whole acrosome has been detected. In cell suspensions stimulated for an acrosome reaction and permeabilized in methanol, $75 \%$ of sperm possessed staining in the acrosomal region, $21 \%$ of sperm was completely unstained and moreover, about 3\% showed IVA-520-binding in the equatorial region of the sperm head (Table 1). IVA520 probably detected molecules on the plasma membrane as well as on structures uncovered by permeabilisation of plasma membrane.

\section{Double staining of sperm with IVA-520 and ACR.4}

In the double antibody staining assay with mAb IVA-520 and ACR.4 (anti-intra-acrosomal protein mAb), four different categories of sperm have been observed. A number of sperm detected in each category are specified in the Table 2. Overall expression of the antigen determined by mAb IVA-520 showed to be different from that detected by mAb ACR.4.

In the sperm populations stimulated for an acrosome reaction we detected approximately $32 \%$ of sperm stained by both antibodies, $36 \%$ of tested cells showed reactivity neither with IVA-520 nor with ACR.4. No cells were positive only for the ACR.4 but, on the other hand, about 33\% (in the staining of frozen thawed sperm about 56\%) reacted with IVA-520 but not with ACR.4 (Fig. 2).This last group of sperm could
Table 2. Double antibody staining of frozen-thawed sperm and sperm stimulated for an acrosome reaction by IVA- 520 and ACR.4

\begin{tabular}{|c|c|c|}
\hline $\begin{array}{l}\text { Reaction pattern } \\
\text { IVA-520/ACR.4 }\end{array}$ & Frozen-thawed & Acrosome-reacted \\
\hline 1 & $39.85 \pm 6.10$ & $31.56 \pm 2.65$ \\
\hline E & $55.88 \pm 5.82$ & $32.50 \pm 2.70$ \\
\hline$\theta$ & - & - \\
\hline$\theta 0$ & $0.41 \pm 0.41$ & $0.32 \pm 0.30$ \\
\hline 00 & $3.78 \pm 1.06$ & $36.30 \pm 5.77$ \\
\hline
\end{tabular}

The values are presented as $\%$ mean \pm SD $(n=20)$. Symbols as in Table 1.

include population of spermatozoa with detached acrosomal content or with unavailable binding site for ACR.4.

\section{Antibody-lectin assay}

The lectin probes (PSA, PNA, WGA, ConA) have been used as a marker of different sperm membranes or acrosomal content, respectively, and considered with the reactivity of mAb IVA-520 (Fig. 3). The percentual abundance of detected sperm populations is described in the Table 3.

\section{PSA, PNA}

Similar reaction patterns of IVA-520, PNA, and PSA were observed. Moreover, no significant differences were detected in the amount of acrosome-reacted spermatozoa using FITC-PSA (34\%) and TRITC-PNA (33\%) or IVA520, respectively (Fig. 3A, B). The positive reactions with both lectins exclude the possibility of detached acrosome (non-reacted with ACR.4); mentioned above, on the contrary, support the hypothesis about unavailable binding site for ACR.4 (intact plasma membrane).

\section{WGA, ConA}

We found out the expression of bovine CD46 only on the sperm positive for WGA (54\%; Fig. 3C). Three categories of

Figure 3. Fluorescence pattern of acrosome-reacted, methanol-permeabilised sperm stained with IVA-520 and lectins (PSA, PNA, WGA, ConA). A. Hoechst 33342 (a), IVA-520+TRITC (b), PSA-FITC (c). B. Hoechst 33342 (a), IVA-520-FITC (b), PNA-TRITC (c). Similar reaction patterns of IVA-520, PNA, and PSA were observed. C. Hoechst 33342 (a), IVA-520-FITC (b), WGA-TRITC (c). CD46 (IVA-520-reactive) molecule was detected only on the sperm positive for WGA. D. Hoechst 33342 (a), IVA-520-FITC (b), ConA-TRITC (c). The population of sperm unstained with IVA-520 but with positive fluorescence signal for ConA in the equatorial region of the sperm head - the inner acrosomal membrane, probably. Captured images were shifted to better resolution of particular colour reaction pattern. 
IVA-520 cell staining have been observed where almost $60 \%$ of sperm showed the consistent reaction pattern as ConA probe. Finally, we observed about $34 \%$ portion of sperm unstained with IVA-520 but with positive fluorescence
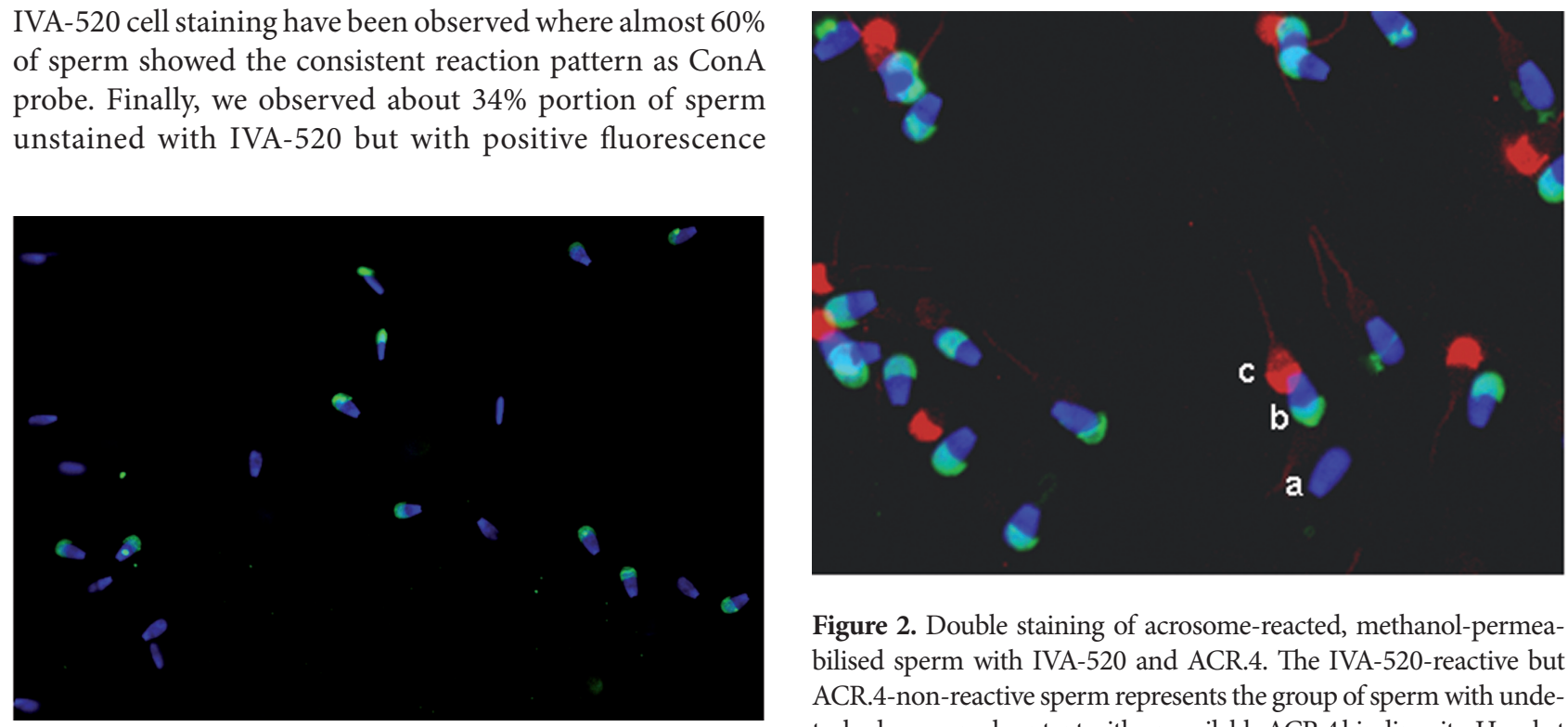

Figure 1. Fluorescence pattern of intact bovine sperm stained with IVA-520. Hoechst 33342 (blue), IVA-520-FITC (green).

Figure 2. Double staining of acrosome-reacted, methanol-permeabilised sperm with IVA-520 and ACR.4. The IVA-520-reactive but ACR.4-non-reactive sperm represents the group of sperm with undetached acrosomal content with unavailable ACR. 4 binding site. Hoechst 33342 (a), IVA-520-FITC (b), ACR.4+TRITC (c). Captured images were shifted to better resolution of particular colour reaction pattern.
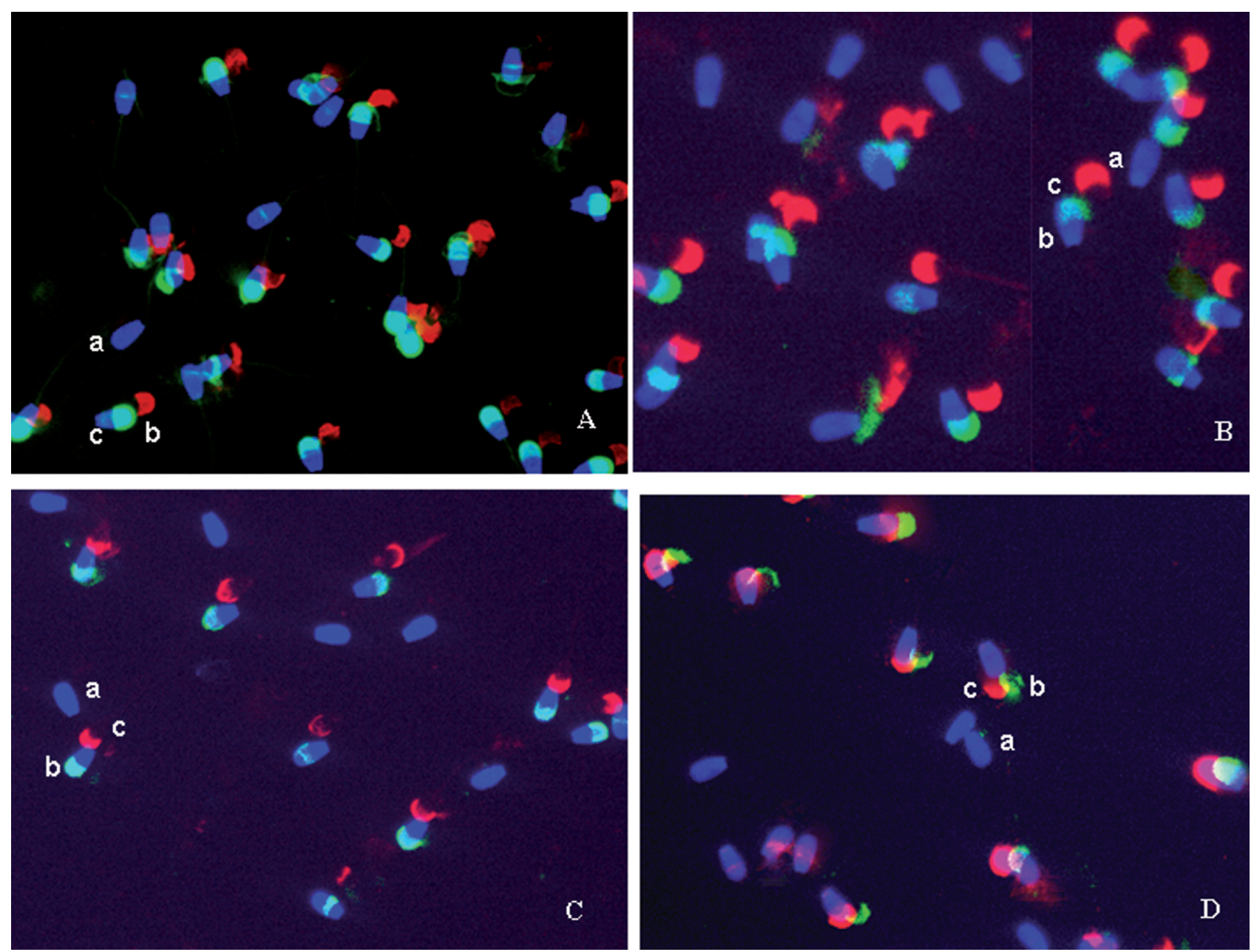
Table 3. Individual fluorescent staining patterns of sperm stimulated for an acrosome reaction treated with IVA-520 and lectins

\begin{tabular}{lcccc}
\hline Reaction pattern & IVA-520/PSA & IVA-520/PNA & IVA-520/WGA & IVA-520/ConA \\
\hline$\theta \ominus$ & $64.89 \pm 4.78$ & $67.28 \pm 1.94$ & $54.74 \pm 1.66$ & $60.85 \pm 6.50$ \\
$\ominus 0$ & $0.65 \pm 0.65$ & - & $1.68 \pm 0.74$ & - \\
0 & $1.37 \pm 1.03$ & - & - & - \\
$\theta \ominus$ & - & $2.84 \pm 2.2$ & $1.68 \pm 0.74$ & $4.73 \pm 1.10$ \\
0 & $7.08 \pm 2.11$ & - & - & $34.41 \pm 5.43$ \\
0 & $26.07 \pm 5.30$ & $29.87 \pm 0.60$ & $41.56 \pm 2.10$ & - \\
\hline
\end{tabular}

The values are presented as $\%$ mean \pm SD $(n=20)$. PSA, Pisum sativum agglutinin; PNA, Arachis hypogaea agglutinin; WGA, Triticum vulgaris agglutinin; ConA, Canavalia ensiformis agglutinin. Symbols as in Table 1.

signal of ConA in the equatorial region of the sperm head (Fig. 3D).

\section{Discussion}

In the first screening experiments higher proportion of IVA-520-reactive sperm was observed (about 43\%) in the permeabilised sperm smears compared with intact sperm. Monoclonal antibody (mAb) IVA-520 detected probably molecules on plasma membrane as well as on structures uncovered by its permeabilisation. Furthermore, reaction pattern of IVA-520 on non-fixed sperm showed to be different from that of mAb ACR.4 against intra-acrosomal protein, released during the acrosome reaction (Pěknicová and Moos 1998). To find out the most probable localization of bovine CD46, the fluorescence antibody-lectin assay was used.

A variety of protocols for the assessment of the binding sites of lectins are available but there are differences between techniques (incubation time, fixation time, fixatives etc.). Moreover, a species-dependent binding specificity of lectins should be taken into consideration.

As a first probe the PSA-FITC conjugate has been involved in the antibody-lectin assay. According to Cross et al. (1986) the residual acrosomal content of human sperm appears to be a major site of PSA binding, on the other hand, Aitken and Brindle (1993) and Farlin et al. (1992) reported that PSA binds to the acrosomal vesicles as they are a result of an acrosomal content dispersal after outer and overlaying plasma membrane swelling and fusion. While the binding site of PSA lectin is localized in the acrosomal content, acrosome-reacted spermatozoa shows no fluorescence signal in the acrosomal portion of the sperm head or its confinement to the equatorial segment as signs of the acrosome loss. The strongly labeled acrosomal region revealed the presence of the acrosome (Cross et al. 1986). In our experiments, generally the majority of the sperm cells showed similar reaction patterns with IVA-520 and PSA, about $30 \%$ of sperm were acrosome reacted.

There are controversial findings and certain species-specific differences in the binding sites of PNA. While Mortimer and coworkers (1987) and Aitken and Brindle (1993) state that PNA binds specifically to the outer acrosomal membrane of human spermatozoa, Carver-Ward et al. (1997) argues that this is more likely to be the inner acrosomal membrane, in a similar manner to CD46. The PNA binding to the outer acrosomal membrane was verified in canine (Sirivaidyapong et al. 2000), boar (Flesch et al. 1998) and stallion (Cheng et al. 1996) sperm. Kitiyanant et al. (2002) reported limited PNA binding to the outer acrosomal membrane of buffalo spermatozoa, too.

In our analyses, similar staining pattern of IVA-520 and PNA has been observed. Moreover, no significant differences were detected in the percentage of acrosome-reacted as well as intact spermatozoa using FITC-PSA (34\%) and TRITCPNA (33\%) or IVA-520 (36\%), respectively. These results support the findings that PNA binds to the outer acrosomal membrane of bovine sperm rather than inner acrosomal membrane and we suppose that IVA-520 recognizes the protein located on the outer acrosomal membrane.

A further lectin, WGA has been involved in our analyses. Gabriel et al. (1995) reported that WGA binds to the inner acrosomal membrane and equatorial segment of human sperm. On the other hand, the strong binding overall surface of human sperm (Fierro et al. 1996), plasma membrane of boar (Flesch et al. 1998), rabbit (Nicolson et al. 1977) and bovine (Medeiros and Parrish 1996) sperm was shown. We found out the expression of bovine CD46 only on the sperm positive for WGA. We suppose that the sperm plasma membrane can be detected in the same time as the outer acrosomal membrane (the permeabilisation of sperm uncovered the outer acrosomal membrane for antibody binding) and the process of the acrosome reaction involves common detachment of the both membranes and they are 
not detectable. Therefore, we were not able to distinguish the binding site of IVA-520 on outer acrosomal membrane from those on plasma membrane. The immunoprecipitation of biotinylated surface antigens of fresh ejaculated sperm with IVA-520 (data not shown) supported the hypothesis about presence of CD46 also on the plasma membrane.

To confirm or exclude expression of CD46 on the inner acrosomal membrane of bovine sperm we treated the acrosome-reacted sperm with IVA-520-FITC and ConA-TRITC. There were indicated binding sites for ConA on both the inner and outer acrosomal membrane and in addition on the plasma membrane of boar sperm (Töpfer-Petersen et al. 1984). Flesch et al. (1998) did not show defined binding patterns of ConA on boar sperm, too. On the other hand, the binding sites for ConA on the inner acrosomal membrane of human (Carver-Ward et al. 1997; Köhn et al. 1997) and bovine sperm (Nishikimi et al. 1997) were reported. We observed about $34 \%$ portion of sperm unstained with IVA520 but with positive fluorescence signal for ConA in the equatorial region of the sperm head - the inner acrosomal membrane, probably.

Finally, the methanol permeabilisation of sperm uncovered other binding sites under the plasma and/or outer acrosomal membrane; therefore, in part of sperm we probably detected the same reaction pattern with IVA-520, lectins and ACR4, respectively. On the contrary, the groups of sperm reacted differently, suggested expression of bovine CD46 on the outer acrosomal membrane and possibly on the plasma membrane of bovine sperm but not on the inner acrosomal membrane and in the acrosomal content.

Acknowledgements. We wish to thank Zuzana Nádaždyová for skillful technical assistance. This work was supported by the VEGA2/0001/09 grant and in part by the APVV-VVCE-0064-07 grant. J. J., J. A., M. S., K. M. and L. H. have no conflict of interest and no financial interest in the publication of this manuscript.

\section{References}

Aitken R. J., Brindle J. P. (1993): Analysis of the ability of three probes targeting the outer membrane or acrosomal contents to detect the acrosome reaction in human spermatozoa. Hum. Reprod. 8, 1663-1669

Anderson D. J., Michalson J. S., Johnson P. M. (1989): Trophoblast/ leukocyte common antigen is expressed by human testicular germ cells and appears on the surface of acrosome-reacted sperm. Biol. Reprod. 41, 285-293 doi:10.1095/biolreprod41.2.285

Anderson D. J., Abbott A. F., Jack R. M. (1993): The role of complement component $\mathrm{C} 3 \mathrm{~b}$ and its receptors in sperm-oocyte interaction. Proc. Natl. Acad. Sci. U.S.A. 90, 10051-10055 doi:10.1073/pnas.90.21.10051

Antalíková J., Simon M., Jankovičová J., Horovská L. (2007): Identification of MCP/CD46 analogue on bovine erythrocytes using the new monoclonal antibody IVA-520. Vet. Immunol. Immunopathol. 115, 155-159

doi:10.1016/j.vetimm.2006.10.013

Ballard L. L., Bora N. S., Yu G. H., Atkinson J. P. (1988): Biochemical characterisation of membrane cofactor protein of the complement system. J. Immunol. 141, 3923-3929

Carver-Ward J. A., Moran-Verbeek J. M., Hollanders J. M.G. (1997): Comparative flow cytometric analysis of the human sperm acrosome reaction using CD46 antibody and lectins. J. Assist. Reprod. Gen. 14, 111-119 doi:10.1007/BF02765780

Cervoni F., Oglesby T. J., Fénichel P., Dohr G., Rossi B., Atkinson J. P., Hsi B. L. (1993): Expression of decay-accelerating factor (CD55) of the complement system on human spermatozoa. J. Immunol. 151, 939-948

Cheng F. P., Fazeh A., Voorhout W. F., Marks A., Bevers M. M., Colenbrander B. (1996): Use of peanut agglutinin to assess the acrosomal status and the zona pellucida-induced acrosome reaction in stallion spermatozoa. J. Androl. 17, 674-682

Clarke G. N, Hsieh C, Koh S. H, Cauchi M. N. (1984): Sperm antibodies, immunoglobulins, and complement in human follicular fluid. Am. J. Reprod. Immunol. 5, 179-181

Cross N. L., Morales P., Overstreet J. W., Hanson F. W. (1986): Two simple methods for detecting acrosome-reacted human sperm. Gamete Research 15, 213-226 doi:10.1002/mrd.1120150303

Farlin M. E., Jusko D. J., Graham J. K., Squires E. L. (1992): Assessment of Pisum sativum agglutinin in identifying acrosomal damage in stallion spermatozoa. Mol. Reprod. Dev. 32, 23-27 doi:10.1002/mrd.1080320105

Fenichel P., Dohr G., Grivaux C., Cervoni F., Donzeau M., Hsi B. L. (1990): Localization and characterization of the acrosomal antigen recognized by GB24 on human spermatozoa. Mol. Reprod. Dev. 27, 173-178

doi:10.1002/mrd.1080270214

Fenichel P., Cervoni F., Hofmann P., Deckert M., Emiliozzi C., Hsi B. L., Rossi B. (1994): Expression of the complement regulatory protein CD59 on human spermatozoa: characterization and role in gametic interaction. Mol. Reprod. Dev. 38, 338-346 doi:10.1002/mrd.1080380316

Fierro R., Foliquet B., Grignon G., Danie, M., Bene M. C., Faure G. C., Barbarino-Monnier P. (1996): Lectin-binding sites on human sperm during acrosome reaction:modifications judged by electron microscopy/flow cytometry. Arch. Androl. 36, 187-196 doi:10.3109/01485019608987095

Flesch F. M., Voorhout W. F., Colenbrander B., van Golde L. M. G., Gadella B. M. (1998): Use of lectins to characterize plasma membrane preparations from boar spermatozoa: A novel technique for monitoring membrane purity and quantity. Biol. Reprod. 59, 1530-1539 doi:10.1095/biolreprod59.6.1530

Gabriel L. K., Franken D. R., Van Den Horst G., Kruger T. F. (1995): Fluorescein isothiocyanate conjugate-wheat germ agglutinin staining of human spermatozoa and fertilization in vitro. Fertil. Steril. 63, 894-901

Hosokawa M., Nonaka M., Okada N., Nonaka M., Okada H. (1996): Molecular cloning of guinea-pig membrane cofactor protein, preferential expression in testis. J. Immunol. 157, 4946-4952 
Inoue N., Ikawa M., Nakanishi T., Matsumoto M., Nomura M., Seya T., Okabe M. (2003): Disruption of mouse CD46 causes an accelerated spontaneous acrosome reaction in sperm. Mol. Cell. Biol. 23, 2614-2622 doi:10.1128/MCB.23.7.2614-2622.2003

Kitiyanant Y., Chisalee B., Pavasuthipaisit K. (2002): Evaluation of the acrosome reaction and viability in buffalo spermatozoa using two staining methods: the effects of heparin and calcium inophore A23187. Int. J. Androl. 25, 215-222 doi:10.1046/j.1365-2605.2002.00350.x

Köhn F. M., Mack S. R., Schill W. B., Zaneveld I. J. D. (1997): Detection of human sperm acrosome reaction: comparison between methods using double staining, Pisum sativum agglutinin, concanavalin A and transmission electron microscopy. Hum. Reprod. 12, 714-721 doi:10.1093/humrep/12.4.714

Makarevich A. V., Markkula M. (2002): Apoptosis and cell proliferation potential of bovine embryos stimulated with insulin- like growth factor I during in vitro maturation and culture. Biol. Reprod. 66, 386-392 doi:10.1095/biolreprod66.2.386

Medeiros C. M. O., Parrish J. J. (1996): Changes in lectin binding to bovine sperm during heparin-induced capacitation. Mol. Reprod. Dev. 44, 525-532 doi:10.1002/(SICI)1098-2795(199608)44:4<525::AID-MRD12>3.0.CO;2-X

Mizuno M., Harris C. L., Johnson P. M., Morgan B. P. (2004): Rat membrane cofactor protein (MCP; CD46) is expressed only in the acrosome of developing and mature spermatozoa and mediates binding to immobilized activated C3. Biol. Reprod. 71, 1374-1383 doi:10.1095/biolreprod.104.030114

Mortimer D., Curtis E. F., Miller R. G. (1987): Specific labeling by peanut agglutinin of the outer acrosomal membrane of the human spermatozoon. J. Reprod. Fertil. 81, 127-135 doi:10.1530/jrf.0.0810127

Nicolson G. L., Uzui N., Yanagimachi R., Yanagimachi H., Smith J. R. (1977): Lectin-binding sites on the plasma membrane of rabbit spermatozoa. J. Cell Biology 74, 950-962 doi:10.1083/jcb.74.3.950

Nishikimi A., Yamada M., Minami N., Utsumi K. (1997): Evaluation of acrosomal status of bovine spermatozoa using concanavalin lectin. Theriogenology 48, 1007-1016 doi:10.1016/S0093-691X(97)00327-0

Oglesby T. J., Allen C. J., Liszewski M. K., White D. J. G., Atkinson J. P. (1992): Membrane cofactor protein (CD46) protects cells from complement-mediated attack by an intrinsic mechanism. J. Exp. Med. 175, 1547-1551 doi:10.1084/jem.175.6.1547

Perricone R., Pasetto N., De Carolis C., Vaquero E., Piccione E., Baschieri L., Fontana L. (1992): Functionally active complement is present in human ovarian follicular fluid and can be activated by seminal plasma. Clin. Exp. Immunol. 89, 154-157 doi:10.1111/j.1365-2249.1992.tb06895.x

Perricone R., De Carolis C., Moretti C., Santuari E., de Sanctis G., Fontana L.(1990): Complement, complement activation and anafylatoxins in human ovarian follicular fluid Clin. Exp. Immunol. 82, 359-362

doi:10.1111/j.1365-2249.1990.tb05453.x

Pěknicová J., Moos J. (1998): Antibody against 28kDa intra-acrosomal sperm protein as a tool for evaluation of acrosomal integrity in bull spermatozoa. Folia Biologica 44, 93-96

Riley R. C, Kemper C., Leung M., Atkinson J. P. (2002): Characterization of human membrane Cofactor protein (MCP; CD46) on spermatozoa. Mol. Reprod. Dev. 62, 534-546 doi:10.1002/mrd.10144

Riley-Vargas R. C, Lanzendorf S., Atkinson J. P. (2005): Targeted and restricted complement activation on acrosome reacted spermatozoa. J. Clin. Invest. 115, 1241-1249

Seya T., Turner J., Atkinson J. P. (1986): Purification and characterization of a membrane protein (gp45-70) which is a cofactor for cleavage of C3b and C4b. J. Exp. Med. 163, $837-855$

doi:10.1084/jem.163.4.837

Seya T., Atkinson J. P. (1989): Functional properties of membrane cofactor protein of complement. Biochem. J. 264, 581-588

Sirivaidyapong S., Cheng F. P., Marks A., Voorhout W. F., Bevers M. M., Colenbrander B. (2000): Effect of sperm diluents on the acrosome reaction in the canine sperm. Theriogenology 53, 789-802 doi:10.1016/S0093-691X(99)00274-5

Töpfer-Petersen E., Januschke E., Schmoeclel C., Schill W. B. (1984): Ultrastructural localization of lectin binding sites of the acrosomal membrane system of boar spermatozoa. Andrologia 16, 539-547 doi:10.1111/j.1439-0272.1984.tb00410.x

Vanderpuye O. A, Labarrere C. A, McIntyre J. A. (1992): The complement system in human reproduction Am. J. Reprod. Immunol. 27, 145-155

Received: October 11, 2010

Final version accepted: December 14, 2010 